\title{
A new ocular motor sign in acute vestibular syndrome: Is HINTS plus enough?
}

\author{
${ }^{1}$ Vishal Pawar DNB Neurology, ${ }^{2}$ Aishwarya Anand, ${ }^{3}$ Prasanna Kulkarni; ${ }^{4} \mathrm{Ji}$ Soo Kim $M D$ PhD \\ ${ }^{1}$ Aster Gardens Specialty clinic, UAE; ${ }^{2}$ MCV ENT hospitals, India $;{ }^{3}$ Aster Medinova diagnostic center, \\ Dubai, UAE; ${ }^{4}$ Department of Neurology, Seoul National University College of Medicine, Seoul, Korea
}

\begin{abstract}
A 66-year-old hypertensive and diabetic male presented with acute vestibular syndrome for three days. HINTS plus examination was performed. The horizontal head impulse test was positive on the left side. Video oculography showed centripetal nystagmus on gaze testing in the dark and test of skew was negative. There was no new hearing loss on the finger rub test. On neurological examination, he had severe postural instability and saccadic smooth pursuit. Radio-imaging studies were conducted to rule out the possibility of stroke. CT brain showed infarction in the territory of the medial branch of the right posterior inferior cerebellar artery. MRI brain confirmed the diagnosis. Thus, posterior circulation stroke can present with acute vestibular syndrome mimicking acute unilateral vestibulopathy. However, the presence of associated neurological symptoms like gait ataxia, centripetal nystagmus and vascular risk factors pointed towards a central cause. Clinical evaluation suggesting a peripheral lesion should never be taken in isolation and needs to be correlated with other associated signs. We describe centripetal nystagmus without fixation as a new oculomotor sign in acute vestibular syndrome.
\end{abstract}

Keywords: Pseudo-Acute Unilateral Vestibulopathy, posterior circulation stroke, cerebellar stroke, positive head impulse test, posterior inferior cerebellar artery infarct, centripetal nystagmus

\section{INTRODUCTION}

Stroke is a common cause of mortality and morbidity. ${ }^{1,2}$ The posterior circulation strokes account for $20 \%$ of all ischemic strokes. ${ }^{3}$ Cerebellar strokes (infarction or haemorrhage) account for $50 \%$ of the posterior circulation strokes. ${ }^{4}$ Symptoms of isolated cerebellar stroke are varied and subtle. Hence it is usually underestimated or missed on clinical evaluation. ${ }^{5}$

Cerebellar stroke is considered as the most frequent cause of vascular 'acute vestibular syndrome' (AVS) manifesting in the form of sudden onset vertigo and dizziness. ${ }^{6}$ Studies have shown that acute vestibular syndrome is presenting manifestation in approximately $25 \%$ of the patients with posterior circulation stroke, especially in the territory of anterior (AICA) and posterior inferior cerebellar artery (PICA). ${ }^{7}$

Central nervous system lesions in the lower brainstem or cerebellum (e.g., infarction of the cerebellar nodulus or flocculus or rostral medullary and pontine lesions $)^{8}$ can mimic acute unilateral vestibulopathy (AUV) and are termed as vestibular pseudo-neuritis (Pseudo-AUV)
Although acute labyrinthine disorders causing AUV are usually benign and self-limiting, vascular injuries of the cerebellum may develop mass effect and can be life-threatening. ${ }^{6}$ In such cases, patient history and bedside clinical examination allows us to differentiate between a peripheral and central lesion. ${ }^{9}$ Presence of vertigo along with neurological signs including cranial nerve findings, hemiparesis, facial weakness, diplopia, hyperesthesia, Horner's Syndrome and gazeevoked nystagmus helps to suspect a central lesion. ${ }^{10} \mathrm{~A}$ three-step bedside oculomotor exam Head-Impulse-Nystagmus-Test-of-skew (HINTS) protocol is considered more sensitive bedside evaluation for the diagnosis of stroke..$^{11}$ Positive head impulse test (HIT) indicates peripheral vestibular lesion over a central lesion. ${ }^{12}$ We are presenting a case of acute vestibular syndrome in which the HINTS plus protocol was in favor of the peripheral lesion (AUV). The presence of other signs like severe postural instability, smooth pursuit abnormalities and centripetal nystagmus without fixation pointed towards the central cause and helped in diagnosis. 


\section{CASE REPORT}

A 66-years-old gentleman with hypertension, diabetes and coronary artery disease came with a history of sudden onset rotatory vertigo associated with nausea and vomiting from three days. Vertigo was spontaneous and was associated with an severe gait ataxia. He denied the history of tinnitus, hearing impairment, numbness of the face, trunk or extremities, dysarthria, dysphagia, diplopia, hiccoughs and loss of consciousness. On examination, the blood pressure was 159/105 mm of $\mathrm{Hg}$. There was no spontaneous or gaze-evoked nystagmus on a bedside ocular motor examination. Bedside head impulse test (HIT) revealed small but consistent corrective catch-up saccade when the head was turned to the left side. There was no skew deviation on alternate cover testing and no new hearing loss on the finger rub test. A CT scan of the brain (Figure 1), showed acute infarction in the territory of the right posterior inferior cerebellar artery, involving the right cerebellar hemisphere, including the cerebellar tonsil. Patchy areas of infarcts were also seen in the medial part of a left cerebellar hemisphere. No infarction was observed in the brainstem.

The MRI brain (Figure 2) obtained after 14 days of the onset of symptoms confirmed the findings of the CT brain. It also showed an additional acute lacunar infarct in the left corona radiata.

The patient was managed on antiplatelet and statins. Sugar levels and blood pressure were controlled with antidiabetic and antihypertensive medications, respectively. After 2 days his vertigo resolved totally, and gait improved significantly. On follow-up after 10 days, the patient recovered

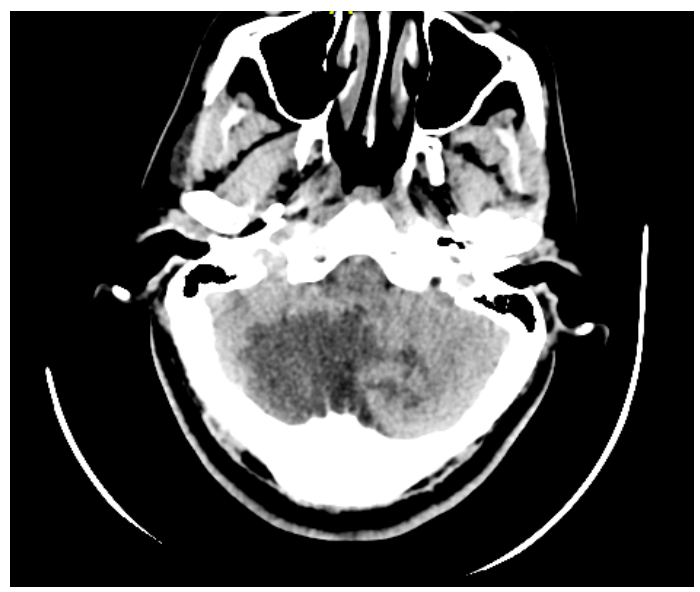

Figure 1. The axial CT scan images demonstrate a large region of hypoattenuation involving the right cerebellar hemisphere as well as patchy areas of infarction in the left cerebellar hemisphere. almost completely.

Bedside HIT was performed manually with a rapid thrust of the head of approximately 15-degree amplitude in the horizontal plane.

\section{Oculography}

The nystagmus, saccades, smooth pursuit was recorded using a video-oculography system (Cyclops, Medtech. Bengaluru, India). The videooculography testing showed normal saccades in both horizontal and vertical planes. The smooth

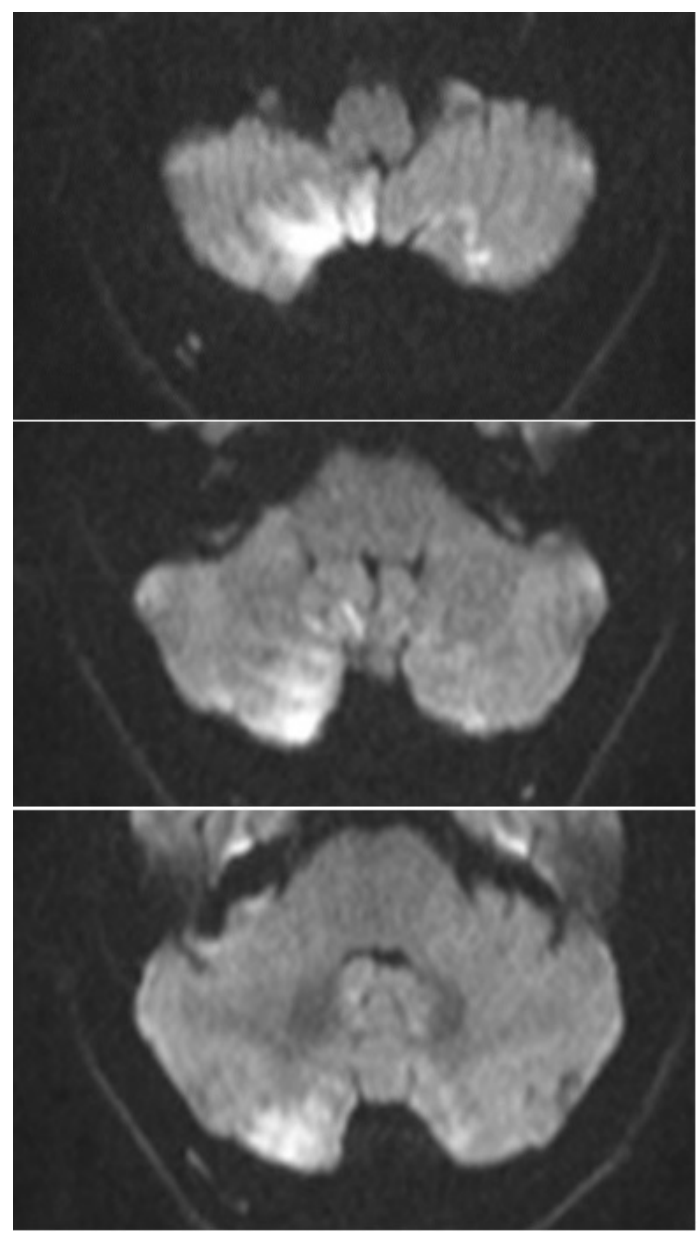

Figure 2. The diffusion-weighted images through the posterior fossa demonstrated restricted diffusion in right cerebellar hemisphere involving the right cerebellar tonsil (Ventral paraflocculus), biventral lobule (Dorsal paraflocculus), gracile lobule and inferior semilunar lobule. There were patchy areas of diffusion hyperintensities within the left biventral lobule (Dorsal paraflocculus), gracile lobule and inferior semilunar lobule - consistent with acute infarct in the territory of the bi-hemispheric right posterior inferior cerebellar artery. 

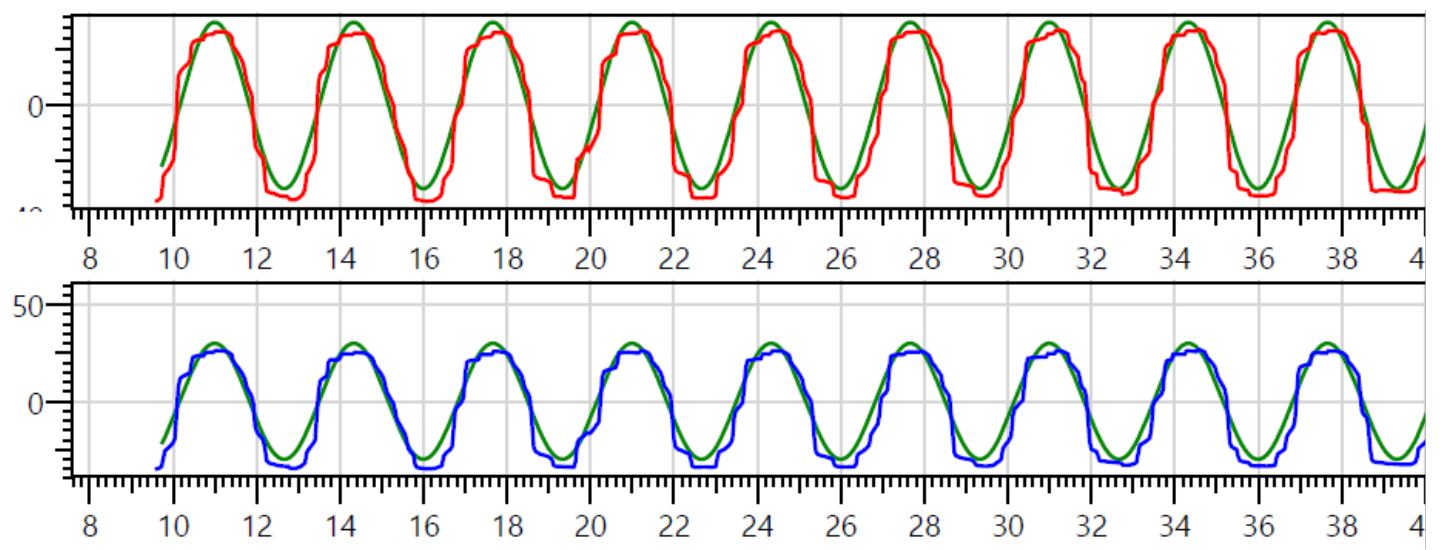

Figure 3. Horizontal smooth pursuit

pursuit was saccadic in both horizontal (Figure 3) and vertical planes (Figure 4). The optokinetic gain was asymmetric and there was a directional preponderance of nystagmus to right and down on optokinetic testing. In the dark (without fixation), the gaze testing showed centripetal nystagmus without fixation (Video 1: Nystagmus beating to right on left gaze and beating to left on right gaze).

Follow up examination of the patient on day 10 of the follow-up showed negative bedside head impulse and disappearance of centripetal nystagmus. The patient was able to walk unsupported.

\section{DISCUSSION}

Dizziness and vertigo are common symptoms in patients presenting to neurology or otolaryngology clinics. ${ }^{6,10}$

The cerebellar nodulus has inhibitory projections to ipsilateral vestibular nucleus..$^{13}$ Considering that the nodulo-vestibular fibers on right side are involved in this patient, the vestibular tone on the right side will increase as compared to the left side due to the lack of inhibition. In turn it mimics left unilateral vestibulopathy which can explain the positive head-impulse test on left side, right beating nystagmus without fixation and pivoting to left side on Unterberger's test performed on Day 6.

About $10 \%-20 \%$ of dizzy patients visit emergency departments (ED) with sudden onset acute vertigo, which is termed as acute vestibular syndrome when associated with nausea, vomiting and head motion intolerance..$^{14,15}$ The most common cause of the acute vestibular syndrome is acute unilateral vestibulopathy of peripheral origin. ${ }^{15,16}$

Literature has documented cases of cerebellar infarction in the territory of the medial branch of the posterior inferior cerebellar artery (mPICA)..$^{17,18}$ PICA is a branch of the vertebral artery, and occasionally it may arise directly from the basilar artery. The course of PICA is usually tortuous, and the territory can be variable. After its origin, it courses transversely and downward around the medulla. It then forms the first caudal loop to supply to the tonsil and second loop to supply to an inferior vermis. It also supplies choroid plexus of the fourth ventricle and inferior surface of cerebellum. ${ }^{17,19}$ Amarenco et al. reported three clinical patterns of cerebellar infarction in PICA territory as 1) Pseudo-labyrinthine

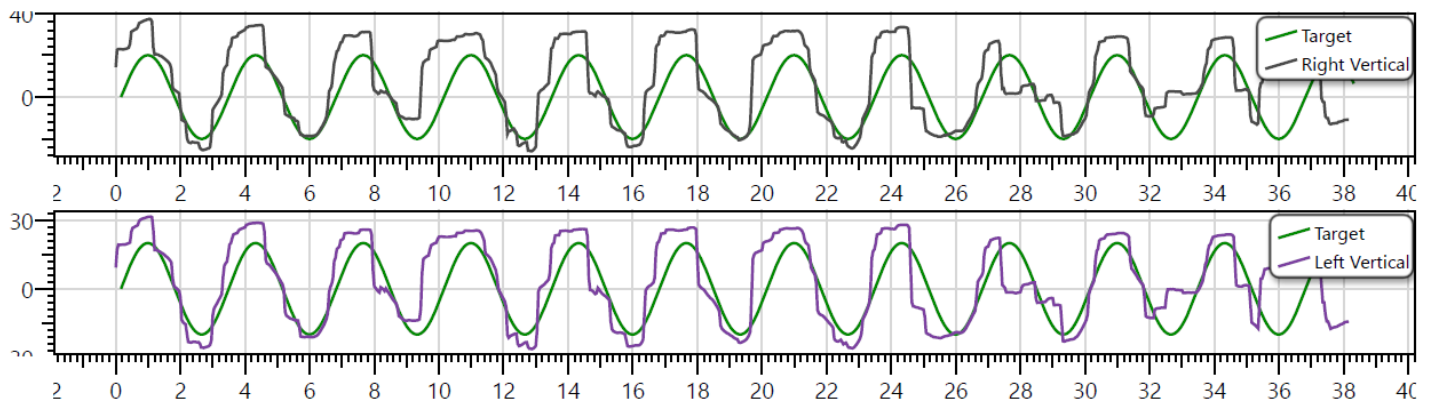

Figure 4. Vertical smooth pursuit 
signs with or without dysmetria and ataxia with sparing of medulla 2) Complete or incomplete Wallenberg's syndrome with involvement of medulla and 3) Silent infarction. ${ }^{17}$

Infarction induced damage to the central vestibular structures, and its connections in the brainstem can give rise to the clinical picture of acute vestibular syndrome. ${ }^{7}$ A three-step bedside oculomotor exam HINTS (Head-ImpulseNystagmus-Test-of-skew) protocol is considered more sensitive bedside evaluation for the diagnosis of stroke in a patient with AUV. ${ }^{11}$ If the patient has a negative head impulse test, alternating fast phase of the nystagmus (Gaze-evoked nystagmus) and re-fixation on a cover test, it is diagnosed as stroke.

Positive head-impulse test (HIT) implies a deficit in the vestibulo-ocular reflex (VOR). ${ }^{10}$ However false-positive HIT was reported in patients with central lesions, e.g. in case of cerebellar $\operatorname{ataxia}^{20}$ and patient with unilateral cerebellar lesion. ${ }^{12}$ Likewise, in a cross-sectional study by Newman-Toker et al., three patients with stroke demonstrated a positive HIT. For vestibulocerebellar stroke, positive HIT was considered as a result of mass effect on the vestibular nucleus or eighthnerve root-entry zone in the pons. In a pontocerebellar stroke, positive HIT was speculated due to labyrinthine infarction. In extensive case of Ponto-cerebello-labyrinthine stroke, a positive HIT was thought due to the vestibular nucleus or eighth nerve root-entry-zone infarction in the lateral pons. ${ }^{11}$ Kheradmad and $Z_{e}{ }^{21}$ reported positive head impulse in isolated floccular infarctions and elucidated the role of the floccular lesion in positive HIT. A lesion in the flocculus could be the cause of positive HIT in our patient. Thus contrary to conventional perception, presence of positive HIT cannot be exclusively relied upon, and additional clinical features must be considered.

The cerebellum plays a significant role in maintaining optimal ocular motor performance. ${ }^{21}$ In healthy individuals, the control systems involved in maintaining steady gaze are the visual fixation, the vestibule-ocular reflex and a gaze-holding system (the neural integrator). Disturbance in any of these control mechanism results in abnormal fixation manifesting in the form of nystagmus and saccadic intrusion or oscillations. ${ }^{22}$ The optokinetic nystagmus is essential for keeping stable retinal image during head motion relative to the environment. Brainstem and cerebellum control this highly reflexive circuits. ${ }^{23}$ Centripetal nystagmus in eccentric gaze, which consists of centrifugally directed slow phases and centripetally directed quick phases, may reflect instability in the gaze-holding networks, similar to the velocityincreasing waveforms of downbeat nystagmus seen in some patients with cerebellar disease. Thus, centripetal nystagmus is explained as an attempt by brainstem or cerebellar mechanism to correct for the centripetal drift of gaze-evoked nystagmus. ${ }^{24}$ Previous studies also supported that lesions of cerebellar structure, including flocculus/ paraflocculus, uvula and vermis could cause disorders of smooth pursuit. ${ }^{25}$ In the present case, the saccadic smooth pursuit in both horizontal and vertical plane suggested a lesion of the flocculus/ paraflocculus that impair smooth tracking of the moving target when the head is in fixed position..$^{21}$ Thus, asymmetric optokinetic gain and centripetal nystagmus in current case hint towards a central lesion.

Diseases affecting brainstem, cerebellum or peripheral vestibular system can present with skew deviation. ${ }^{26}$ However, in a cross-sectional study by Newman-Toker et al. out of 69 confirmed cases of stroke by imaging 51 showed the absence of skew deviation. ${ }^{27}$ These findings suggest that skew deviation is a specific but not a sensitive marker of the central lesion, and its absence can be misleading in stroke. Likewise, ataxia, impaired coordination of voluntary muscle movement are common physical findings of cerebellar dysfunction..$^{28}$

Thus in our case, initial clinical examination suggested a possibility of peripheral lesion due to a positive HIT, absence of skew deviation and absence of new hearing loss. However, the presence of severe postural instability, centripetal nystagmus without fixation, saccadic smooth pursuit, inability to fixate with saccadic intrusions and vascular risk factors pointed towards a central cause.

In conclusion, posterior circulation stroke can present with acute vestibular syndrome mimicking acute unilateral vestibulopathy with positive head impulse test. However, the presence of other neurological signs like a saccadic smooth pursuit, severe postural instability, plus the ocular motor sign - centripetal nystagmus without fixation, can help in the early diagnosis of a central lesion. (video 1, https://neurology-asia.org/content/26/ 4/neuroasia-2021-26(4)-853-v1.mp4)

\section{DISCLOSURE}

All experiments followed the tenets of the Declaration of Helsinki. The patient consented 
for the use of the videos for the educational and research purpose. The study was approved by Institutional ethics committee.

\section{REFERENCES}

1. Katan M, Luft A. Global burden of stroke. Semin Neurol 2018; 38:208-11.

2. Kalache A, Aboderin I. Stroke: the global burden. Health Policy Plan 1995; 10:1-21.

3. Nouh A, Remke J, Ruland S. Ischemic posterior circulation stroke: a review of anatomy, clinical presentations, diagnosis, and current management. Front Neurol 2014; 5:Article 30.

4. Ng Z, Yang W, Seet E, et al. Cerebellar strokes : a clinical outcome review of 79 cases. Singapore Med $J$ 2015; 56:145-9.

5. Nickel A, Cheng B, Pinnschmidt $\mathrm{H}$, et al. Clinical outcome of isolated cerebellar stroke-a prospective observation study. Front Neurol 2018; 9:580-3.

6. Lee H. Neuro-otological aspects of cerebellar stroke syndrome. J Clin Neurol 2009; 5:65-73.

7. Choi J, Kim H, Choi K. Isolated vestibular syndrome in posterior circulation stroke. Neurol Clin Pract 2014; 4:410-8

8. Choi KD, Lee H, Kim JS. Vertigo in brainstem and cerebellar strokes. Curr Opin Neurol 2013; 26:90-5.

9. Strupp M, Magnusson M. Acute unilateral vestibulopathy. Neurol Clin 2015; 33:669-85.

10. Armato E, Ferri E, Pinzani A, Ulmer E. Cerebellar haemorrhage mimicking acute peripheral vestibulopathy: the role of the video head impuse test in differential diagnosis. Acta Otorhinolaryngol Ital 2014; 34:288-91.

11. Newman-Toker D, Kattah J, Talkad A, Wang D, Hsieh Y. H.I.N.T.S to diagnose stroke in the acute vestibular syndrome - Three-step bedside oculomotor exam more sensitive than early MRI DWI. Stroke 2009; 40:3504-10.

12. Baek S, Choi J, Jung J, et al. Abnormal head impulse test in a unilateral cerebellar lesion. J Clin Neurol 2015; 11:279-82.

13. Meng H, Blazquez PM, Dickman JD, et al. Diversity of the vestibular nuclei neurons targeted by cerebellar nodulus inhibition. J Physiol 2014; 592:171-88.

14. Tarnutzer A, Berkowitz A, Robinson K, et al. Does my dizzy patient have a stroke? A systematic review of bedside diagnosis in acute vestibular syndrome. CMAJ 2011; 183:E571-92.

15. Hotson J, Baloh R. Acute vestibular syndrome. $N$ Engl J Med 1998; 339:680-5.

16. Kattah J, Talkad A, Wang D, Hsieh Y, NewmanToker D. HINTS to diagnose stroke in the acute vestibular syndrome: three-step bedside oculomotor examination more sensitive than early MRI diffusionweighted imaging. Stroke 2009; 40:3504-10.

17. Amarenco P, Roullet E, Hommel M, Chaine P, Marteau R. Infraction in the territory of the medial branch of the posterior inferior cerebellar artery. $J$ Neurol Neurosurg Psychiatry 1990; 53:731-5.

18. Charles N, Froment C, Rode G, et al. Vertigo and upside down vision due to an infract in the territory of the medial branch of the posterior inferior cerebellar artery caused by dissection of a vertebral artery. $J$ Neurol Neurosurg Psychiatry 1992; 55:188-9.

19. Toms J, Wadhwa R, Ambekar S, Cuellar H. Bihemispheric posterior inferior cerebellar artery occurring with an azygos anterior cerebral artery: case study. Case Rep Radiol 2014:541081.

20. Kremmyda O, Kirchner H, Glasauer S, et al. Falsepositive Head -Impulse test in test cerebellar ataxia. Front Neurol 2012; 3:162-8.

21. Kheradmad A, Zee D. Cerebellum and ocular motor control. Front Neurol 2011; 2:53-68.

22. Abadi R. Mechanisms underlying nystagmus. $J R$ Soc Med 2002; 95:231-4.

23. Ruehl R, Hinkel C, Bauermann T, Eulenburg P. Delineating function and connectivity of optokinetic hubs in the cerebellum and the brainstem. Brain Struct Funct 2017; 222:4163-85.

24. Leech J, Greshy M, Hess K, et al. Gaze failure, drifting eye movements ,and centripetal nystagmus in cerebellar disease. Br J Ophthalmol 1977; 61:774-81.

25. Baier B, Stoeter P, Dieterich M. Anatomical correlates of ocular motor deficits in cerebellar lesion. Brain 2009; 132:2114-24.

26. Wong A. Understanding skew deviation and a new clinical test to differentiate it from trochlear nerve palsy. J AAPOS 2010; 14:61-7.

27. Newman-Toker D, Kattah J, Alvernia J, Wang D. Normal head impulse test differentiates acute cerebellar strokes from vestibular neuritis. Neurology 2008; 70:2378-85.

28. Ashizawa T, Xia G. Ataxia. Continuum (Minneap Minn) 2016; 22:1208-26. 